\title{
The evolution of carbon and oxygen in the bulge and disk of the Milky Way
}

\author{
G. Cescutti ${ }^{1}$, F. Matteucci ${ }^{1,2}$, A. McWilliam ${ }^{3}$, and C. Chiappini ${ }^{2,4}$ \\ 1 Dipartimento di Astronomia, Universitá di Trieste, via G.B. Tiepolo 11, 34131 Trieste, Italy \\ e-mail: cescutti@oats.inaf.it \\ 2 INAF Osservatorio Astronomico di Trieste, via G.B. Tiepolo 11, 34131 Trieste, Italy \\ 3 Observatories of the Carnegie Institution of Washington, 813 Santa Barbara St., Pasadena, CA 91101, USA \\ ${ }^{4}$ Observatoire Astronomique de l’Université de Genève, 51 Chemin des Mailletes, Sauverny, 1290, Switzerland
}

Received 24 June 2009 / Accepted 22 July 2009

\section{ABSTRACT}

\begin{abstract}
Context. The evolution of $\mathrm{C}$ and $\mathrm{O}$ abundances in the Milky Way can impose strong constraints on stellar nucleosynthesis and help in understanding the formation and evolution of our Galaxy.

Aims. The aim of this paper is to review the measured $\mathrm{C}$ and $\mathrm{O}$ abundances in the disk and bulge of the Galaxy and compare the results to predictions of Galactic chemical evolution models.

Methods. We adopt two successful chemical evolution models for the bulge and the disk, respectively. They assume the same nucleosynthesis prescriptions but different histories of star formation.

Results. The data show a clear distinction between the trend of $[\mathrm{C} / \mathrm{O}]$ in the thick and thin Galactic disks, while the thick disk and bulge trends are indistinguishable with a large $(>0.5 \mathrm{dex})$ increase in the $[\mathrm{C} / \mathrm{O}]$ ratio in the range from -0.1 to $+0.4 \mathrm{dex}$ for $[\mathrm{O} / \mathrm{H}]$. In our models we consider yields from massive stars with and without the inclusion of metallicity-dependent stellar winds. The observed increase in the $[\mathrm{C} / \mathrm{O}]$ with metallicity in the bulge and thick disk lies between the predictions utilizing mass-loss rates of Maeder and Meynet \& Maeder. A model without metallicity-dependent yields completely fails to match the observations. Thus, the relative increase in carbon abundance at high metallicity appears to come from metallicity-dependent stellar winds in massive stars. These results also explain the steep decline of the $[\mathrm{O} / \mathrm{Fe}]$ ratio with $[\mathrm{Fe} / \mathrm{H}]$ in the Galactic bulge, while the $[\mathrm{Mg} / \mathrm{Fe}]$ ratio is enhanced at all $[\mathrm{Fe} / \mathrm{H}]$.

Conclusions. We conclude that data and models are consistent with a rapid bulge and thick disk formation timescales, and with metallicity-dependent yields for $\mathrm{C}$ and $\mathrm{O}$. The observed too high $[\mathrm{C} / \mathrm{O}]$ ratios at low metallicity in the bulge may stem from an unaccounted source of carbon: very fast rotating metal poor stars, or metal-poor binary systems whose envelopes were stripped by Roche lobe overflow.
\end{abstract}

Key words. Galaxy: evolution - Galaxy: bulge - Galaxy: disk - Galaxy: abundances - stars: abundances nuclear reactions, nucleosynthesis, abundances

\section{Introduction}

A basic contradiction exists in the interpretation of $[\mathrm{O} / \mathrm{Fe}]$ and $[\mathrm{Mg} / \mathrm{Fe}]$ trends in the Galactic bulge (McWilliam \& Rich 2004; Fulbright et al. 2007). The $[\mathrm{O} / \mathrm{Fe}]$ ratio declines steeply with increasing $[\mathrm{Fe} / \mathrm{H}]$, while the $[\mathrm{Mg} / \mathrm{Fe}]$ ratio is enhanced in all bulge stars, and only declines slightly by $[\mathrm{Fe} / \mathrm{H}] \sim+0.5$ dex. A paradigm (e.g. Tinsley 1979; Greggio \& Renzini 1983; Matteucci \& Greggio 1986) often used to explain the alphaelement trend with metallicity, asserts that the $[\mathrm{O} / \mathrm{Fe}]$ ratio declines with $[\mathrm{Fe} / \mathrm{H}]$ due to the addition of $\mathrm{Fe}$ from type Ia supernovae ( $\mathrm{SNe}$ ) on long timescales. Since $\mathrm{Mg}$ and $\mathrm{O}$ are thought to be produced in similar mass (the most massive) progenitors of type II SNe (e.g. Woosley \& Weaver 1995, henceforth WW95), it is expected that $[\mathrm{O} / \mathrm{Fe}]$ and $[\mathrm{Mg} / \mathrm{Fe}]$ should exhibit the same trend with $[\mathrm{Fe} / \mathrm{H}]$. Thus, within this alpha-element paradigm, the $[\mathrm{O} / \mathrm{Fe}]$ trend suggests a longer formation timescale for the bulge than does the $[\mathrm{Mg} / \mathrm{Fe}]$ ratio. McWilliam \& Rich (2004) suggest that the steep decline in $[\mathrm{O} / \mathrm{Fe}]$ actually comes from metallicitydependent winds, and is related to the Wolf-Rayet phenomenon, rather than to the addition of Fe from type Ia SNe.

In a previous paper (McWilliam et al. 2008), we analyzed the trend of the $[\mathrm{O} / \mathrm{Mg}]$ ratio in Galactic disk and bulge stars. The observational data indicated that the $[\mathrm{O} / \mathrm{Mg}]$ showed the same decline with increasing $[\mathrm{Mg} / \mathrm{H}]$ in both the bulge and disk, despite the very different formation timescales of these two systems, and qualitatively consistent with metallicity-dependent $\mathrm{O}$ yields. In the McWilliam et al. (2008) paper we included yields for oxygen from massive stars with metallicity-dependent winds in detailed chemical evolution models for the bulge and disk of the Milky Way. In particular, we adopted the Maeder (1992) yields for massive stars including mass loss; these yields are a strong function of the stellar mass for stars at solar metallicity and above. For masses larger than $25 M_{\odot}$, the oxygen production is strongly depressed as a consequence of this mass loss. In the presence of a strong mass loss during the main sequence phase of massive stars, a larger amount of $\mathrm{He}$ and $\mathrm{C}$ are lost relative to stars evolving at constant mass. This fact produces two main effects: i) a larger production of $\mathrm{He}$ and $\mathrm{C}$ from stars with high metal content; ii) a smaller production of oxygen, due to lack of $\mathrm{C}$ which is lost instead of being transformed into O. In Maeder (1992) and Meynet \& Maeder (2002) this yield effect is important only for the $Z=0.02$ models, while the yields of $\mathrm{C}$ and $\mathrm{O}$ from massive stars with metallicities of $Z=0.004$, and below, do not differ substantially from the yields computed without mass loss (e.g. Woosley \& Weaver 1995). This is due to the fact that 
mass loss in massive stars is a strong function of metallicity. In McWilliam et al. (2008) we showed that the trends of $[\mathrm{O} / \mathrm{Mg}]$, $[\mathrm{Mg} / \mathrm{Fe}]$ and $[\mathrm{O} / \mathrm{Fe}]$ in Galactic bulge and disk stars can be well reproduced by models with the metallicity-dependent yields of Maeder (1992). If these metallicity-dependent yields from massive stars are correct, then the expectation is that the oxygen decline to higher metallicity stars in the bulge, as shown by $[\mathrm{O} / \mathrm{Mg}]$ and $[\mathrm{O} / \mathrm{Fe}]$ trends, should be accompanied by an increase in the $[\mathrm{C} / \mathrm{O}]$ ratio. Thus, the $[\mathrm{C} / \mathrm{O}]$ ratio in the bulge provides a test for the conclusions of McWilliam et al. (2008). We note that since $\mathrm{C}$ and $\mathrm{O}$ are thought to be produced only during hydrostatic phases of stellar evolution the $[\mathrm{C} / \mathrm{O}]$ versus $[\mathrm{O} / \mathrm{H}]$ trend in the Galaxy should be considerably simpler to predict than $[\mathrm{C} / \mathrm{Fe}]$ and $[\mathrm{O} / \mathrm{Fe}]$, because iron is produced in the hard to model explosions of type Ia and type II SNe in quantities that are not well predicted. Indeed, even the theoretical yields for $\mathrm{Mg}$ from type II SNe (e.g. from WW95) are not well predicted and it has been necessary for modelers to employ semi-empirical yields from Galactic chemical evolution models (e.g. François et al. 2004).

In this paper we analyze the effects of stellar yields with mass loss on carbon and $\mathrm{C} / \mathrm{O}$ ratios, both in the bulge and the disk. As mentioned before, the decreased $\mathrm{O}$ production results in an increased $\mathrm{C}$ production and this effect needs to be tested with the observational data. Besides the Maeder (1992) yields, we have included in the chemical evolution models $\mathrm{C}$ and $\mathrm{O}$ yields from massive stars by Meynet \& Maeder (2002), computed with a lower rate of mass loss but including rotation; yields for $Z=0.02$ stars, which were not given in the original paper, were taken from Chiappini et al. (2003b). Yields for super-solar metallicity stars are not available.

The paper is organized as follows: in Sect. 2 we describe the observational data, in Sect. 3 we recall the main assumptions of the adopted chemical evolution models, in Sect. 4 we describe the adopted stellar yields, in Sect. 5 we show the results for the bulge and the disk of the Milky Way. Finally, in Sect. 6 some conclusions are drawn.

\section{Observational data}

We wish to compare the observed trends of $[\mathrm{C} / \mathrm{O}]$ with $[\mathrm{O} / \mathrm{H}]$ in the Galactic thin and thick disks and bulge with predictions of chemical evolution models including yields from stars with metallicity-dependent winds. For the relatively hot unevolved turn-off and main sequence stars carbon and oxygen abundances are most reliably determined from the forbidden [O I] and [C I] lines at $6300 \AA$ and $8727 \AA$, respectively, because these lines have negligible non-LTE abundance corrections. This is unlike the allowed C I and O I transitions with large predicted non-LTE corrections, the estimated values of which have varied significantly (e.g. Fabbian et al. 2006, 2009). The insensitivity of the forbidden lines to non-LTE effects is partly due to the long lifetimes of the upper levels, which allows collisions to set the populations to their local thermodynamic values. However, a cause for care and concern is that the forbidden [C I] and [O I] lines are rather weak in the turnoff and dwarf stars, with equivalent widths typically in the range 2-12 $\mathrm{m} \AA$. Also, only in the last few years it has been appreciated (Allende Prieto et al. 2001) that the [O I] line at $6300 \AA$ is blended with a Ni I line; and that the $[\mathrm{C} \mathrm{I}]$ feature contains a weak Fe I blend (e.g. Bensby \& Feltzing 2006) and lies in the wing of a stronger Si I line. We note, in passing, that the largely ignored [O I] line at $6363 \AA$ is much weaker than the line at $6300 \AA$ and often gives discordant results, which we presume is related to the fact that it lies

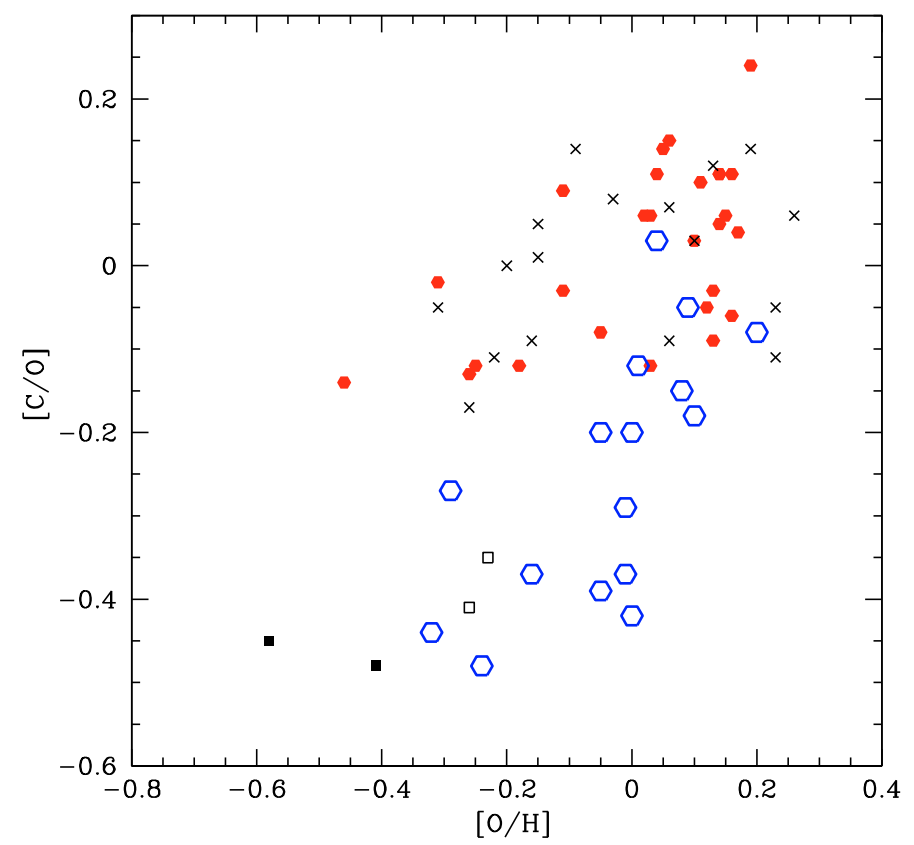

Fig. 1. $[\mathrm{C} / \mathrm{O}]$ ratio versus $[\mathrm{O} / \mathrm{H}]$ for dwarf and turnoff stars in the solar vicinity, based on forbidden $[\mathrm{O} \mathrm{I}]$ and $[\mathrm{C} \mathrm{I}]$ lines, which are insensitive to non-LTE effects. The thin disk stars from corrected Nissen \& Edvardsson (1992), Gustafsson et al. (1999) and Andersson \& Edvardsson (1994), indicated by black crosses are in good agreement with the thin disk results of Bensby \& Feltzing (2006, red filled hexagons). Thick disk stars from Bensby \& Feltzing (2006) are indicated with blue open hexagons; two thick disk stars from Nissen shown with open black squares are consistent with the Bensby \& Feltzing (2006) results. Two halo stars from Nissen (private communication) are shown as filled black squares. This plot shows a clear distinction between the $[\mathrm{C} / \mathrm{O}]$ ratios in the thin and thick disks: the thick disk $[\mathrm{C} / \mathrm{O}]$ ratios are significantly lower than for the thin disk toward lower $[\mathrm{O} / \mathrm{H}]$.

in the middle of a broad $\mathrm{Ca}$ I auto-ionization feature which depresses the central [O I] line depth; most studies fail to take the Ca I auto-ionization feature into account, resulting in spurious oxygen abundance results.

In this paper we employ forbidden line carbon and oxygen abundances of thin and thick disk dwarf/turnoff stars from two main sources: Bensby \& Feltzing (2006) and the corrected oxygen abundances of Nissen \& Edvardsson (1992) and carbon abundances from Gustafsson et al. (1999) and Andersson \& Edvardsson (1994). Nissen (private communication) kindly supplied the revised oxygen abundances, from the [O I] $6300 \AA$ line, corrected for the Ni I blend (which was not a known problem in 1992). While Nissen did not publish the corrected oxygen abundance values they do appear in Fig. 9 of Nissen et al. (2002). We have performed calculations which roughly confirm Nissen's corrections. In Nissen's private communication to us he also included abundances for two extra, unpublished, halo stars.

In Fig. 1 we show literature results of $[\mathrm{C} / \mathrm{O}]$ versus $[\mathrm{O} / \mathrm{H}]$ for the solar vicinity, based on the forbidden [O I] and [C I] lines. The plot shows a clear distinction between the $[\mathrm{C} / \mathrm{O}]$ ratios in the thin and thick disks; the thick disk is characterized by lower $[\mathrm{C} / \mathrm{O}]$ values, which decrease with metallicity, and seem to link to the low $[\mathrm{C} / \mathrm{O}]$ ratio seen in the two halo stars. The good agreement between the Nissen data and those of Bensby \& Feltzing (2006) is encouraging. We note that the population identifications are based on statistical probability, so a small number of misidentifications are possible. The differences in $[\mathrm{C} / \mathrm{O}]$ versus $[\mathrm{O} / \mathrm{H}]$ for the thick and thin disks show that it was not possible 
to make the thick disk from present day thin disk material (e.g. by dynamical heating of the thin disk), or to make the lower metallicity thin disk from thick disk material (see also Chiappini 2009, and Chiappini et al., in preparation for a discussion of the different evolution between the thin and thick disks). Of course, carbon and oxygen abundance measurements are also possible for red giant stars. An advantage is that the [O I] $6300 \AA$ line is stronger in red giants $(\sim 30-70 \mathrm{~m} \AA)$ than dwarf/turnoff stars; the $8727 \AA[\mathrm{C} \mathrm{I}]$ line is also stronger in red giants (e.g. $11 \mathrm{~m} \AA$ in Arcturus), but it has not been used much in these stars. A significant disadvantage posed by the red giants is that stellar evolution effects, during the first dredge-up phase, cause their atmospheres to be altered from their original CNO composition. In the first dredge-up the atmospheres are contaminated by material that previously experienced proton-burning by $\mathrm{CN}$ cycle core burning (e.g. Iben 1964, 1967; Becker \& Iben 1979). Typically, solar neighborhood red giant atmospheres show $\sim 0.2$ dex carbon depletions, but 1 dex depletions are also found (e.g. Lambert \& Ries 1981). However, because most of the missing carbon is turned into nitrogen the original carbon abundance can be estimated from the sum of $\mathrm{C}+\mathrm{N}$. This is a useful trick for measuring the primordial carbon abundance in red giants, and especially important for studying the Galactic bulge $\mathrm{C} / \mathrm{O}$ trend, since the bulge dwarfs are too faint for routine high resolution abundance studies $^{1}$; thus, red giant stars provide the main probe to effectively explore the Galactic bulge $\mathrm{C} / \mathrm{O}$ trend.

In Fig. 2 we show the thin disk $[\mathrm{C} / \mathrm{O}]$ trend with $[\mathrm{O} / \mathrm{H}]$ based on the abundances from dwarf and turnoff stars from Bensby \& Feltzing (2006, red filled hexagons) and Nissen (private communication, red crosses). In addition to these results from dwarf and turnoff thin disk stars we also include $[\mathrm{C} / \mathrm{O}]$ ratios based on the $[(\mathrm{C}+\mathrm{N}) / \mathrm{O}]$ values in the red giants of Mishenina et al. (2006, black filled squares); to do this we sum the $\mathrm{C}$ and $\mathrm{N}$ abundances but we also subtract an estimate of the original $\mathrm{N}$ abundance, based on the solar $\mathrm{N} / \mathrm{Fe}$ ratio, which amounts to almost 0.10 dex. Figure 2 shows that the three sources of $[\mathrm{C} / \mathrm{O}]$ versus $[\mathrm{O} / \mathrm{H}]$ in the thin disk are consistent with a single trend, and a small decrease in $[\mathrm{C} / \mathrm{O}]$, of about $0.2 \mathrm{dex}$, from $[\mathrm{O} / \mathrm{H}]=0.0$ to -0.4 dex. We note that the Mishenina et al. (2006) red giant data lack points at the low $[\mathrm{O} / \mathrm{H}]$ end of the diagram; this probably arises from an absence of carbon abundances for their metalpoor disk stars, due to non-detection of the $\mathrm{C}_{2}$ molecular lines, which they used as a carbon abundance indicator. These molecular $\mathrm{C}_{2}$ lines are relatively weak at solar metallicity, and decrease rapidly in strength to lower metal content. In addition to the decrease in $[\mathrm{C} / \mathrm{O}]$ at low $[\mathrm{O} / \mathrm{H}]$ there may be a decrease in $[\mathrm{C} / \mathrm{O}]$ at the highest metallicity $([\mathrm{O} / \mathrm{H}] \geq+0.15 \mathrm{dex})$, but this conclusion is sensitive to outliers in the plot. If we use only the current carbon abundances for the Mishenina et al. (2006) red giants, without the correction for the $\mathrm{CN}$ cycle transformation of carbon to nitrogen, the thin disk red giants are not consistent with the trend established by the two sources of $[\mathrm{C} / \mathrm{O}]$ ratios for the thin disk dwarf stars, but instead shows depleted [C/O] values by $\sim 0.2$ dex. We take this difference as evidence that our practice of combining the $\mathrm{C}+\mathrm{N}$ abundances, minus the estimated original $\mathrm{N}$, for red giant stars provides sound results, and should give reliable $[\mathrm{C} / \mathrm{O}]$ ratios when applied to the Galactic bulge red giants. We note, however, that the $[\mathrm{C} / \mathrm{O}]$ versus $[\mathrm{O} / \mathrm{H}]$ trend for thin disk red giant stars in Melendez et al. (2008, henceforth M08) show

\footnotetext{
${ }_{1}$ Recently, Johnson et al. (2008) have measured C/O in a single lensed bulge dwarf star spectrum, but the oxygen abundance relied on the highly excited allowed transitions at $7770 \AA$, which are known to suffer from non-LTE effects.
}

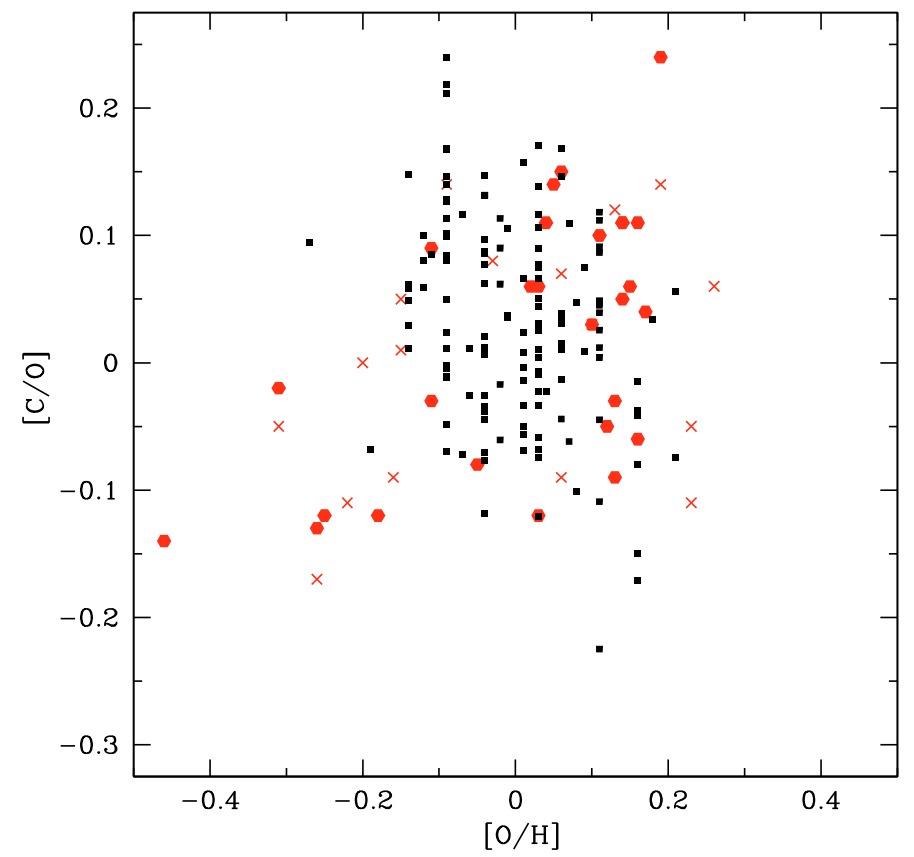

Fig. 2. $[\mathrm{C} / \mathrm{O}]$ versus $[\mathrm{O} / \mathrm{H}]$ for thin disk dwarf/turnoff stars based on forbidden line abundances, from Bensby \& Feltzing (2006, red filled hexagons) and corrected Nissen (1992, red crosses). [C/O] versus [O/H] for thin disk red giant stars are from Mishenina et al. (2006, black filled squares), but corrected for first dredge-up by adding $\mathrm{C}+\mathrm{N}$ (minus the original $\mathrm{N}$ based on the solar $\mathrm{N} / \mathrm{Fe}$ ratio) to estimate the primordial carbon abundance. Note the good agreement between dwarf/turnoff stars, which are unaffected by first dredge-up, and the red giant $[\mathrm{C} / \mathrm{O}]$ ratios from $\mathrm{C}+\mathrm{N}-\mathrm{Fe} \cdot(\mathrm{N} / \mathrm{Fe})_{\odot}$.

larger scatter and a $<0.1$ dex downward shift compared to the dwarf results of Bensby \& Feltzing (2006). However, the M08 results for the thick disk are in good agreement with the Bensby \& Feltzing (2006) values, but with somewhat larger scatter.

For the bulge $[\mathrm{C} / \mathrm{O}]$ data we considered abundance results from Fulbright et al. (2007), M08, and the oxygen abundances from Zoccali et al. (2006) with carbon results for the same stars given in the companion paper by Lecureur et al. (2007). Results from the small overlapping studies of Cunha \& Smith (2006) and Ryde et al. (2009) could also be included in the comparisons. We omit the abundance results for bulge $\mathrm{M}$ giants from Rich \& Origlia (2005) and Rich et al. (2007) since they did not measure nitrogen abundances, required to estimate the primordial carbon.

McWilliam, Fulbright \& Rich (2009, in progress, henceforth MFR09) have recomputed oxygen abundances, for stars in the FMR07 study. In their molecular equilibrium calculations they employed the carbon and nitrogen abundances determined from IR spectra in the same set of stars by M08 (of the 19 bulge stars in M08 18 were studied by FMR07). The MFR09 abundances are an improvement over FMR07, in part because the FMR07 oxygen abundances were computed using approximate $\mathrm{C} / \mathrm{Fe}$ and $\mathrm{N} / \mathrm{Fe}$ abundance ratios, expected for typical red giant stars, rather than measured values. The revised MFR09 O/Fe ratios were based on line-by-line differential abundances relative to the sun, using the [O I] $6300 \AA$ line and five transitions of Fe II.

The differential method employed by MFR09 removed the $g f$ values from the calculation of the $[\mathrm{O} / \mathrm{H}]$ and $[\mathrm{Fe} / \mathrm{H}]$ abundances. Furthermore, because formation of the Fe II lines is similar to the $[\mathrm{O} \mathrm{I}]$ lines, errors in parameters that effect the electron density in the stellar atmospheres (e.g. gravity and $\alpha / \mathrm{Fe}$ ratio) 
have a negligible effect on the $[\mathrm{O} / \mathrm{Fe}]$ ratio determined using these lines. We note that Zoccali et al. (2006) did not discuss the use of Fe II lines or appropriate alpha-enhanced model atmospheres in the determination of their $[\mathrm{O} / \mathrm{Fe}]$ ratios, because they used Fe I lines for the $[\mathrm{O} / \mathrm{Fe}]$ ratio. If so, their $[\mathrm{O} / \mathrm{Fe}]$ ratios would be strongly dependent on the gravity, metallicity and $\alpha / \mathrm{Fe}$ ratios of the input model atmospheres; indeed, their [C/O] versus $[\mathrm{O} / \mathrm{H}]$ trend shows a much larger scatter than the trend found here. Therefore, we respectfully choose not to employ the Zoccali et al. (2006) $[\mathrm{O} / \mathrm{Fe}]$ ratios. For the $[\mathrm{Fe} / \mathrm{H}]$ abundances MFR09 retained the FMR07 $[\mathrm{Fe} / \mathrm{H}]$ values, which were based on differential Fe I line abundances relative to the nearby red giant $\alpha$ Boo, and provide $[\mathrm{Fe} / \mathrm{H}]$ values with small internal scatter.

We note that the $[\mathrm{O} / \mathrm{Fe}]$ versus $[\mathrm{Fe} / \mathrm{H}]$ trend of MFR09 and that of M08 agree very well from $-0.8<[\mathrm{Fe} / \mathrm{H}]<+0.5 \mathrm{dex}$, but below $[\mathrm{Fe} / \mathrm{H}] \sim-0.8$ dex MFR09 $[\mathrm{O} / \mathrm{Fe}]$ values are larger than M08 by $\sim 0.1$ dex. It is likely that the two OH lines in the M08 spectra are relatively weak at these lower metallicities, while the [O I] lines in FMR07/MFR09 are easily measured. Differences between the M08 points and MFR09 values are partly due to the adopted $[\mathrm{Fe} / \mathrm{H}]$ abundance; we do not know the cause of the difference between FMR07/MFR09 and M08 [O/Fe] trends at low metallicity. Perhaps the reduced strength of the Fe I lines, or the small number of detected Fe I lines, in the near-IR spectra of the most metal-poor stars in M08 resulted in less accurate Fe abundances. We also note that the optical [O I] lines in FMR07 are insensitive to non-LTE effects, while $\mathrm{OH}$ lines are sensitive to non-LTE, which is more significant for metal-poor stars. For these reasons we prefer to employ the $[\mathrm{O} / \mathrm{Fe}]$ values computed by MFR09 with the M08 [C/Fe] and $[\mathrm{N} / \mathrm{Fe}]$ ratios.

As mentioned previously, the $\mathrm{C}+\mathrm{N}-\mathrm{Fe} \cdot(\mathrm{N} / \mathrm{Fe})_{\odot}$ provides a good estimate of the original carbon abundance in red giant stars that have experienced first dredge-up of CN processed material. For the bulge results we employ $[\mathrm{O} / \mathrm{Fe}]$ from MFR09 and the $[\mathrm{C} / \mathrm{Fe}]$ and $[\mathrm{N} / \mathrm{Fe}]$ abundances from M08 to determine the primordial $[\mathrm{C} / \mathrm{O}]$ and we use the MFR09 $[\mathrm{O} / \mathrm{Fe}]$ with FMR07 $[\mathrm{Fe} / \mathrm{H}]$ ratios to compute $[\mathrm{O} / \mathrm{H}]$.

In Fig. 3 we show our adopted bulge trend of primordial $[\mathrm{C} / \mathrm{O}]$ versus $[\mathrm{O} / \mathrm{H}]$, estimated from the measured carbon and nitrogen abundances of M08 and the MFR09 [O/FeII] ratios, from the FMR07 EWs. For the $[\mathrm{O} / \mathrm{H}]$ values we add the FMR07 $[\mathrm{Fe} / \mathrm{H}]$ abundances to the robust $[\mathrm{O} / \mathrm{FeII}]$ ratios of MFR09. For comparison, the figure also includes the data for thin and thick disk stars presented in Fig. 1. Figure 3 shows a relatively tight correlation of $[\mathrm{C} / \mathrm{O}]$ versus $[\mathrm{O} / \mathrm{H}]$ in the bulge, with a plateau near $[\mathrm{C} / \mathrm{O}]=-0.50$ dex for metallicities below $[\mathrm{O} / \mathrm{H}] \sim 0$, but rising, roughly linearly from $[\mathrm{O} / \mathrm{H}] \sim-0.1$, with increasing metallicity to $[\mathrm{C} / \mathrm{O}] \sim+0.1-0.2$ dex near $[\mathrm{O} / \mathrm{H}] \sim+0.3$ dex. The most obvious feature is that the bulge $[\mathrm{C} / \mathrm{O}]$ versus $[\mathrm{O} / \mathrm{H}]$ trend is remarkably similar to that of the thick disk, and quite different than the thin disk. We note that the 5 stars from Cunha \& Smith (2006) and the 3 stars from Ryde et al. (2009), most of which are in FMR07 and M08, show similar $[\mathrm{C} / \mathrm{O}]$ versus $[\mathrm{O} / \mathrm{H}]$ to those seen in the bulge in Fig. 3, but with larger scatter than our data. However, there simply aren't enough points in either of these two studies for a useful comparison. Two of the stars in Ryde et al. (2009) are in Cunha \& Smith (2006), but for one (BW IV$329)$ their abundances differ significantly. In addition, the Ryde et al. (2009) [C/O] and [O/H] values for BW IV-203 place it far from the remaining points in Fig. 3, due to its enormous nitrogen abundance; also, FMR07 determined that this star was not a member of the bulge by comparing photometric and ionization based gravities, as stated in that paper. Given the much larger sample of stars in MFR09 and M08, the small scatter evident in

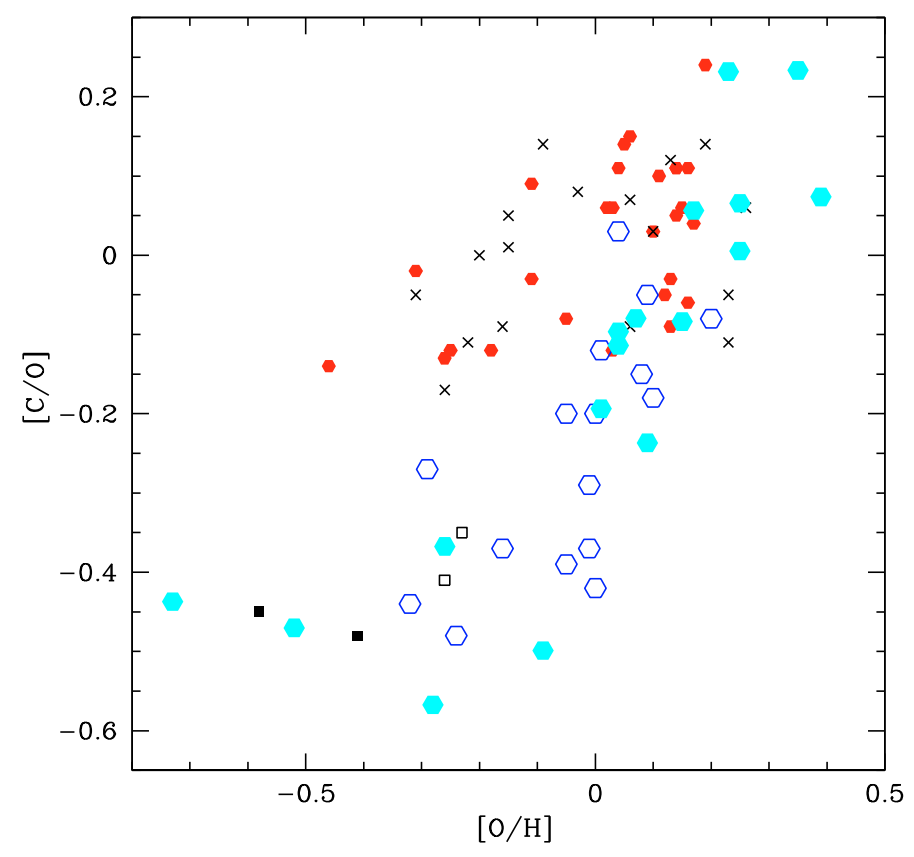

Fig. 3. Our estimated primordial $[\mathrm{C} / \mathrm{O}]$ versus $[\mathrm{O} / \mathrm{H}]$ trend in Galactic bulge red giants (cyan filled hexagons), determined from FMR07, MFR09 and M08, compared with the thin disk (red filled hexagons and black crosses) and thick disk (blue open hexagons and black open squares) dwarf/turnoff stars (identical to Fig. 1). The primordial values for the bulge red giants were estimated from $(\mathrm{C}+\mathrm{N})-\mathrm{Fe} \cdot(\mathrm{N} / \mathrm{Fe})_{\odot}$. The tight correlation of $[\mathrm{C} / \mathrm{O}]$ versus $[\mathrm{O} / \mathrm{H}]$ in the bulge appears identical to the relationship seen in the thick disk stars.

the bulge [C/O] ratios of Fig. 3, the use of the forbidden [O I] line here, and the uncertain systematics of the Ryde et al. (2009) and Cunha \& Smith (2006) results we, respectfully, prefer not to use the latter.

\section{The chemical evolution models}

For studying the chemical evolution of the solar neighborhood, we adopted the model of François et al. (2004), which is an implemented version of the original model by Chiappini et al. (2003a). This model assumes that the Galaxy formed by means of two main accretion episodes, one giving rise to the halo and thick disk and the other forming the thin disk. The infalling gas is always assumed to be of primordial composition. Detailed nucleosynthesis from low and intermediate mass stars, type Ia and type II SNe is taken into account. The IMF is taken from Scalo (1986). For details of this model see François et al. (2004).

For the bulge, we adopted the model of Ballero et al. (2007a), which assumes a rapid formation timescale, of $0.3-0.5 \mathrm{Gyr}$, from gas accumulated during the halo collapse. The efficiency of star formation (star formation per unit mass of gas) is 20 times higher (i.e. $\left.20 \mathrm{Gyr}^{-1}\right)$ than in the solar vicinity $\left(1 \mathrm{Gyr}^{-1}\right)$. The IMF is flatter than in the solar vicinity, as required by the observed bulge stellar metallicity distribution: we assumed the following IMF: $x=0.95$ for stars with $m>1 M_{\odot}$, and $x=0.33$ for stars with $m \leq 1 M_{\odot}$ (see Ballero et al. 2007a, for details of this model).

\section{Nucleosynthesis prescriptions}

In this work we investigate 3 different prescriptions for the yields from massive stars, in particular: i) the metal dependent yields of Woosley \& Weaver (1995, hereafter WW95); ii) the metal 
dependent stellar yields from massive stars of Meynet \& Maeder (2002, hereafter MM02), which take the effects of mass loss and rotation on stellar evolution into account, used also in Chiappini et al. (2003b) and Carigi et al. (2005); iii) the yields with mass loss and no rotation of M92. It is worth noting that both the M92 and MM02 yields from massive stars include mass loss by stellar winds, but the MM02 calculations adopt a significantly lower rate of mass loss. The recent mass loss rates are factors of 2 to 3 times lower than previously adopted by M92 and they include stellar rotation.

For low to intermediate mass stars we use in all the models the van den Hoek \& Groenewegen (1997) metallicity dependent yields (in particular the tables assuming a mass loss parameter, $\eta$, varying with metallicity). We also tested the Karakas \& Lattanzio (2007) metallicity dependent yields of C and $\mathrm{O}$ from low and intermediate mass stars, and found negligible differences in the results compared to those obtained with the van den Hoek \& Groenewegen (1997) yields. We then adopted the latter ones for consistency with our previous paper (McWilliam et al. 2008).

We note that the more recent oxygen yields of Hirschi et al. (2005) are significantly higher than MM02, by factors of 2-3. In fact, Hirschi et al. (2005) stellar evolution calculations were carried out with different input parameters (e.g. different amounts of overshooting). Moreover they only included material lost in the wind, and nucleosynthesis up to the pre-SN stage; they did not include any treatment of the supernova explosion, explosive nucleosynthesis calculations (e.g. explosive $\mathrm{O}$ burning), or other issues related to SN nucleosynthesis yields, as discussed in WW95 and many other works. Therefore, we avoid combining these different set of yields which otherwise would produce an artificial metallicity dependency of the oxygen yields.

The M92 and MM02 yields did not perform supernova nucleosynthesis calculations, but they employed the Arnett (1991) relationship between $\mathrm{O}$ yield and the $M_{\alpha}$ core mass to determine their oxygen yields. The Arnett (1991) O yield relationship with $M_{\alpha}$ is very similar to the independent result of WW95.

For $\mathrm{Mg}$ we employ in all the models the semi-empirical yields for SN II and SNIa computed by François et al. (2004) to have the best agreement in the solar vicinity.

\section{Results}

We run several models for both the bulge and the solar vicinity with yields from massive stars taken from various authors: A) metallicity-dependent WW95 yields, without mass-loss; B) WW95 yields for metallicities below solar and the M92 yields (with mass loss) for $Z \geq Z_{\odot}$; C) WW95 yields for metallicities lower than solar and MM02 yields (including mass-loss) for $Z \geq Z_{\odot}$; D) MM02 metal-dependent yields (with mass loss) only.

In Fig. 4 we show the results of four chemical evolution models compared with the solar neighborhood $[\mathrm{C} / \mathrm{H}]$ versus $[\mathrm{O} / \mathrm{H}]$ trends for the thin and thick disks. A strength of this plot is that only $\mathrm{C}$ and $\mathrm{O}$ are involved, so complications from the production of Fe in type Ia and Type II supernovae are avoided. Because carbon and oxygen are produced during hydrostatic phases of stellar evolution the yields are much more tractable than for Fe.

The predictions for the thin disk $[\mathrm{C} / \mathrm{O}]$ trend show an increase in the $[\mathrm{C} / \mathrm{O}]$ ratio associated with carbon production from relatively low mass stars, on long timescales ( $>1 \mathrm{Gyr}$ ). This partly agrees with Bensby \& Feltzing (2009) conclusion, who argued that the production of carbon occurred on a similar timescale to the production of $\mathrm{Fe}$, based on a flat $[\mathrm{C} / \mathrm{Fe}]=0.0$ trend for both the thin and thick Galactic disks below solar

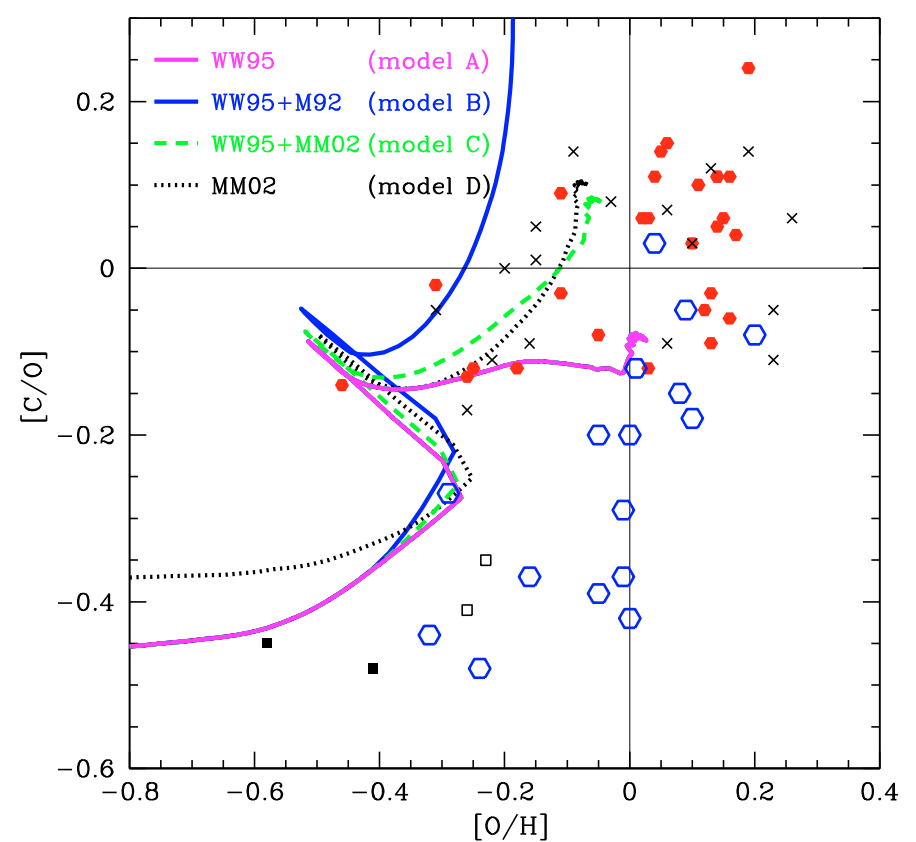

Fig. 4. Predictions of $[\mathrm{C} / \mathrm{O}]$ versus $[\mathrm{O} / \mathrm{H}]$ in the thin disk compared to measured abundances in the solar vicinity. See Fig. 1 for the key to the data symbols. We present the results of four different chemical evolution models, with and without metallicity-dependent yields. To better match the $[\mathrm{C} / \mathrm{O}]$ versus $[\mathrm{O} / \mathrm{H}]$ slope in the bulge (cf. Fig. 5) we interpolate MM02 and M92 yields between $Z=0.004$ and $Z=0.002$.

$[\mathrm{Fe} / \mathrm{H}]$. Our interpretation based on the data and our predictions in Fig. 4 is that the thin disk shows higher [C/O] ratios than the thick disk, due to increased carbon yields from low mass stars; our predictions show such increased carbon yields on long time scales (from low mass stars) for the thin disk only. Our thin disk models suggest that approximately $50 \%$ of the carbon produced along the whole galactic history comes from low mass stars (see also Carigi et al. 2005).

We presume that, similar to the bulge, the thick disk evolved more rapidly than the thin disk (see also Melendez et al. 2008), given the similarity of the thick disk and bulge $[\mathrm{C} / \mathrm{O}]$ versus $[\mathrm{O} / \mathrm{H}]$ trend in Fig. 3. Therefore, the thick disk would not be expected to have included the extra carbon produced on long time scales by low mass stars; this is in contrast to the conclusion of Bensby \& Feltzing (2009).

Several features in Fig. 4 are worthy of note: first, the slope of the $[\mathrm{C} / \mathrm{O}]$ versus $[\mathrm{O} / \mathrm{H}]$ trend in the thin disk is best matched by models $\mathrm{C}$ and $\mathrm{D}$, which employ the MM02 yields with metaldependent stellar winds plus rotation. The higher mass-loss rates used in the M92 metal-dependent winds result in too steep a $[\mathrm{C} / \mathrm{O}]$ versus $[\mathrm{O} / \mathrm{H}]$ slope, as if too much carbon is released at the expense of oxygen. On the other hand model A, which employed the metal-dependent evolution of WW95 but ignored mass loss from stellar winds and rotation, gave too shallow a slope in the $[\mathrm{C} / \mathrm{O}]$ versus $[\mathrm{O} / \mathrm{H}]$ relation for the thin disk.

We draw attention to the inflection points in our thin disk models in Fig. 4, where $[\mathrm{O} / \mathrm{H}]$ appears to decrease temporarily. This is due to the competition between the star formation rate (SFR) and the gas infall rate. In fact, in the two infall model a gap in the SF is naturally produced at the end of the halo-thick disk formation. Such a gap is mainly due to the different infall episodes coupled with a threshold in the gas density for SF (see also a discussion of this point in Chiappini et al. 2003b). At the inflection point, star formation is not active because the infalling 
gas forming the thin disk has still a density below the threshold. Therefore, $\mathrm{O}$ is no more produced while the thin disk is growing by means of $\mathrm{H}$ infalling gas. At later times, on long timescales, the disk $[\mathrm{O} / \mathrm{H}]$ increases again, due to the activation of $\mathrm{SF}$ and to the diminishing effect of the declining infall rate. Also, the $[\mathrm{C} / \mathrm{O}]$ ratio increases further due to carbon from long-lived stars.

The maximum $[\mathrm{O} / \mathrm{H}]$ and $[\mathrm{C} / \mathrm{O}]$ ratios reached by our models is also worth noting: Fig. 4 shows a progression in the maximum $[\mathrm{O} / \mathrm{H}]$ from the models that decreases with increasing stellar wind mass-loss rate, while the $[\mathrm{C} / \mathrm{O}]$ ratio increases with increasing stellar mass-loss rates (WW95 < MM02 < M92). These maximum ratios are simply due to the increase in carbon yield, at the expense of oxygen, as the mass-loss prescription is increased. Clearly, none of our models reaches the highest $[\mathrm{O} / \mathrm{H}]$ values observed in the thin disk. The solution to this lacuna is not obvious: perhaps the theoretical nucleosynthesis yields need to be increased (e.g. due to nuclear reaction rates or stellar evolution effects), or we should include in the models the migration of more metal rich stars in the solar vicinity (see Schönrich \& Binney 2009). Ideally, our predictions should match both the $[\mathrm{C} / \mathrm{O}]$ trend with $[\mathrm{O} / \mathrm{H}]$ and the $[\mathrm{O} / \mathrm{H}]$ distribution function, similar to the metallicity distribution function (MDF) from $[\mathrm{Fe} / \mathrm{H}]$ frequently used to constrain the gas infall rate parameter.

It is encouraging that the thin disk $[\mathrm{C} / \mathrm{O}]$ observations nicely overlap our predictions, at least for $[\mathrm{O} / \mathrm{H}]$ less than solar, but zero-point shifts due to the exact value of the solar oxygen and carbon abundances could slightly diminish this pleasing agreement; Asplund's group have recently suggested an increased solar oxygen abundance, by 0.05 dex (Scott et al. 2009). Other zero-point shifts may occur if theoretical yields are over/under estimated, or sources omitted; however, differences between predicted trends between the thin disk and bulge/thick will be more robust.

In Fig. 5 we show the $[\mathrm{C} / \mathrm{O}]$ versus $[\mathrm{O} / \mathrm{H}]$ data and predictions from our bulge models. We note that for the models that employ the MM02 and M92 yields we have interpolated the metallicity-dependent yields between $Z=0.02$ and $Z=0.004$. We believe this to be a reasonable procedure, but if this interpolation is not adopted then the predicted $[\mathrm{C} / \mathrm{O}]$ trends rise nearly vertically, much steeper than the gentle positive slope described by the data; we have employed this same interpolation of yields over metallicity for the thin disk models previously described. We first note that the predictions with the metallicity-dependent yields from WW95 completely fail to match the bulge observations, while the shape of the observed upward trend in $[\mathrm{C} / \mathrm{O}]$ with increasing $[\mathrm{O} / \mathrm{H}]$ seems to fall between the predictions based on the MM02 and MM92 yields affected by metallicitydependent winds in massive stars. We take these predictions as strong evidence that yields from massive stars with metallicitydependent winds are largely responsible for the increase in observed trend of $[\mathrm{C} / \mathrm{O}]$ and the decrease in observed trends of $[\mathrm{O} / \mathrm{Mg}]$ (and $[\mathrm{O} / \mathrm{Fe}]$ ) in the bulge. Thus, the apparent inconsistency between the enhanced $[\mathrm{Mg} / \mathrm{Fe}]$ ratios and declining $[\mathrm{O} / \mathrm{Fe}]$ with $[\mathrm{Fe} / \mathrm{H}]$ in the bulge is simply a metallicity effect, as suggested by McWilliam et al. (2008). Apparently, the metallicitydependent winds do not decrease the $\mathrm{Mg}$ yields as much as the $\mathrm{O}$ yields. It is worth noting that for the thin disk the increase in the $[\mathrm{C} / \mathrm{O}]$ trend is mainly due to the contribution of intermediate and low mass stars at low $[\mathrm{O} / \mathrm{H}]$ because of the low star formation rate in the thin disk, whereas the increase of $[\mathrm{C} / \mathrm{O}]$ trend in the bulge is mainly due to the $\mathrm{C}$ produced by massive stars occuring at a high $[\mathrm{O} / \mathrm{H}]$ because of the fast star formation rate in the bulge. In other words, the different star formation rates in the

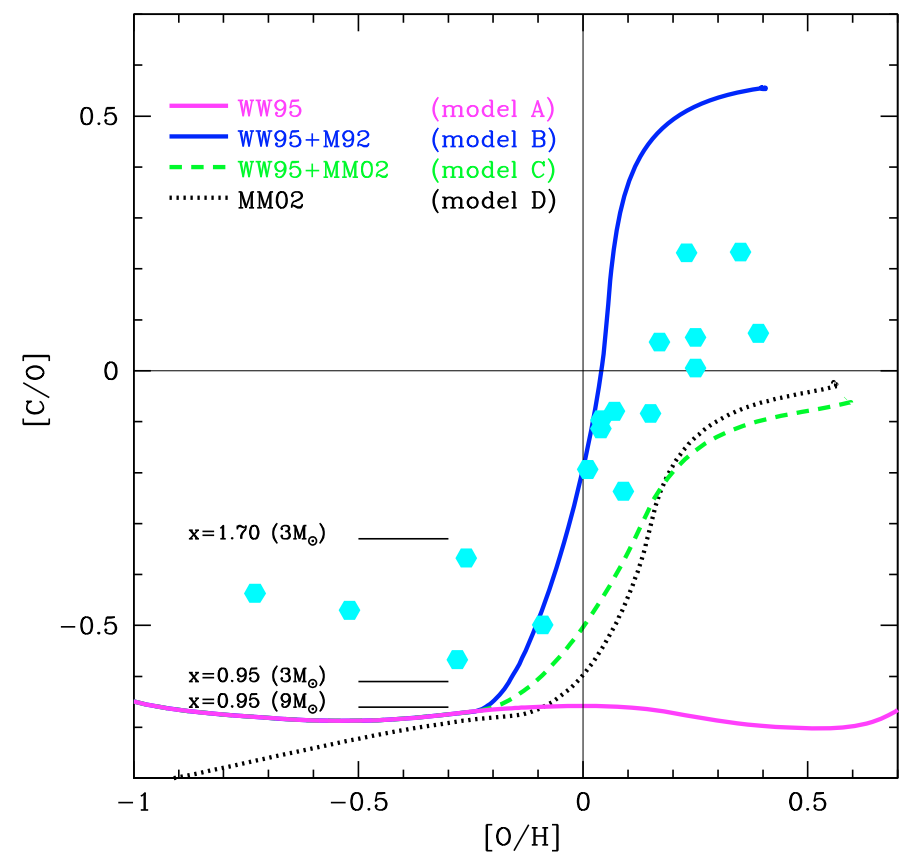

Fig. 5. Model predictions compared to observed $[\mathrm{C} / \mathrm{O}]$ versus $[\mathrm{O} / \mathrm{H}]$ in the Galactic bulge. Model A (red solid line) shows the expected trend for WW95 yields, which did not include metallicity-dependent massloss. Model B (blue solid line) shows our predictions with WW95 yields at low metallicity but M92 yields for solar metal content. The M92 yields include the effects of metallicity-dependent winds at relatively high mass-loss rate, but did not include rotational effects. Model C (green dashed line) shows the result when the lower mass-loss rates, but including rotation, of MM02 are included, together with the WW95 yields for low metallicity. Model D (black dotted line) employs only the MM02 yields which include metallicity-dependent winds and rotation.

bulge and thin disk produce quite different $[\mathrm{O} / \mathrm{H}]$ - age relationships, with the bulge reaching very fast high $[\mathrm{O} / \mathrm{H}]$ values.

Closer inspection of Fig. 5 shows that below $[\mathrm{O} / \mathrm{H}] \sim$ -0.2 dex the $[\mathrm{C} / \mathrm{O}]$ ratio in the bulge is roughly constant, near -0.5 dex. This differs from the predicted $[\mathrm{C} / \mathrm{O}]$ level near -0.7 dex. To explain the difference between the observed and predicted $[\mathrm{C} / \mathrm{O}]$ ratio in the metal-poor bulge one might appeal to the bulge IMF slope for massive stars, because more massive stars have lower $[\mathrm{C} / \mathrm{O}]$ yield ratios, due to the larger core masses. However, the bulge IMF slope has been set by Ballero et al. (2007a) at $x=0.95$ in order to explain the combination of a near-solar mean metallicity distribution function and the high $[\mathrm{Mg} / \mathrm{Fe}]$ (which suggests a rapid formation timescale). Thus the bulge IMF, which Ballero et al. (2007a) require to be skewed to more massive stars than the disk, can only be significantly altered if the bulge did not form rapidly (contrary to the $[\mathrm{Mg} / \mathrm{Fe}]$ evidence). The black horizontal lines marked in Fig. 5 show the $[\mathrm{C} / \mathrm{O}]$ ratios for two IMF slopes: $x=0.95$, the Ballero et al. (2007a) bulge value, and $x=1.70$, the Kroupa (2002) value for the solar neighborhood (corrected for binaries). The masses in parentheses indicate the lower mass limit of the IMF integrations. A $9 M_{\odot}$ limit shows the $[\mathrm{C} / \mathrm{O}]$ ratio produced by type II supernovae only, while the $3 M_{\odot}$ limit indicates the lowest mass star that could have died during the bulge formation time $(<1 \mathrm{Gyr})$. Thus, to explain the bulge $[\mathrm{C} / \mathrm{O}]$ plateau at lower $[\mathrm{O} / \mathrm{H}]$ would require an IMF slope of approximately $x=1.3$.

Other possibilities that could increase the predicted $[\mathrm{C} / \mathrm{O}]$ in the low metallicity bulge are: 1 ) the $\mathrm{C}$ production from very fast rotating extremely metal poor stars, but in this case a plateau in $[\mathrm{C} / \mathrm{O}]$ would not be obtained but rather an initial peak followed 


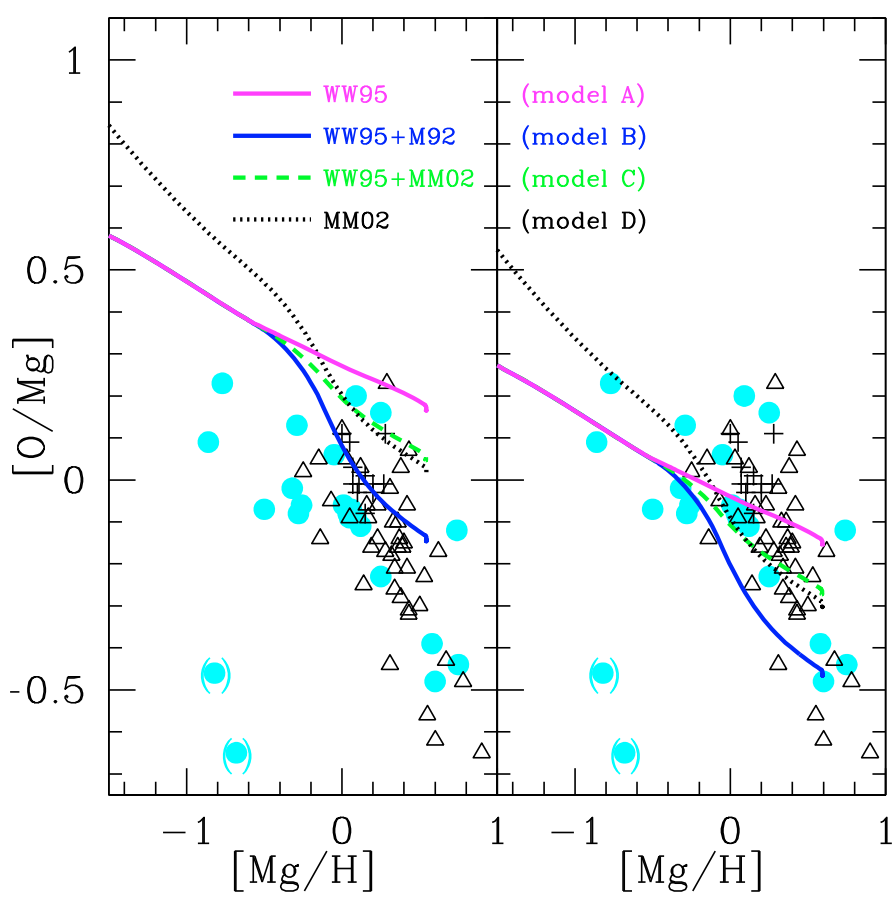

Fig. 6. Comparison between the predictions of our 4 models for $[\mathrm{O} / \mathrm{Mg}]$ vs. $[\mathrm{Mg} / \mathrm{H}]$ and the observations in the bulge. The observational data for the bulge are: the filled circles are the data from MFR09 and FMR07; the open triangles are by Lecureur et al. (2007); the plus signs are the infrared results from Rich \& Origlia (2005). Note that the two bulge stars in parenthesis (from FMR07) show the effect of proton burning products in their atmospheres, therefore, they have probably suffered a reduction in the envelope oxygen abundances via stellar evolution, so their oxygen abundances do not reflect the bulge composition. Left panel: the model results are normalized to Asplund et al. (2005) solar abundances. Right panel: the model results are normalized to the solar abundances of Grevesse \& Sauval (1998).

by a further decrease (see Chiappini et al. 2006), although in a starburst situation, such as in ellipticals or bulges, fast stellar rotators could have been present up to larger metallicities, as recently suggested by Pipino et al. (2009); 2) a decrease in the ${ }^{12} \mathrm{C}(\alpha, \gamma){ }^{16} \mathrm{O}$ rate, although we are unable to give a quantitative estimate or say whether this can reasonably be the case; 3 ) an extra source of carbon at low metallicity could be provided by low metallicity massive binaries. We recall that an early suggestion for the production of Wolf-Rayet (WR) stars was through Roche lobe mass-loss/stripping of the outer envelopes of massive stars (Paczyński 1967). While this mechanism is not favored as the main source of WR stars in the Magellanic Clouds and Galaxy, it should have occurred at some level, even in low metallicity massive binaries. Given that the stripping of the envelopes of massive stars by winds at solar metallicities is responsible for the increased carbon and decreased oxygen yields, it seems possible that metal-poor WR stars formed through Roche-lobe mass loss in binary systems would also have increased carbon and decreased oxygen yields. Quantitative calculations are required to determine whether this mechanism could have produced the observed $[\mathrm{C} / \mathrm{O}]$ ratio in metal-poor bulge stars. We assume that at high metallicity the Roche lobe stripping of metal-poor massive binary star envelopes is pre-empted by the effects of metallicitydependent winds, so this particular extra source of carbon probably mainly affects the metal-poor regime. Finally, we should take into account that another option to explain the disagreement between predictions and observations is represented by the uncertainties in the observational data.
In Fig. 6 we show the $[\mathrm{O} / \mathrm{Mg}]$ vs. $[\mathrm{Mg} / \mathrm{H}]$ in the bulge. This figure is similar to the one in McWilliam et al. (2008) with the addition of the predictions of models $C$ and $D$ not present in that paper. Another difference with the same figure in McWilliam et al. (2008) is that here we adopted interpolated yields, as explained before. This makes the $[\mathrm{O} / \mathrm{Mg}]$ ratio to decline less abruptly. We note that, apart from a zero point problem, the best slope for the $[\mathrm{O} / \mathrm{Mg}]$ ratio for $[\mathrm{Mg} / \mathrm{H}]>0$ is still obtained with the M92 yields (model B), but models $\mathrm{C}$ and can still be acceptable. Concerning the zero point, as already discussed in our previous paper, the agreement with observations depends also on the assumed solar abundances: here we have normalized all models to the Asplund et al. (2005) abundances. If we had assumed Grevesse \& Sauval (1998) normalization, as in Ballero et al. (2007a), the predicted [O/Mg] would decrease by $\sim 0.2$ dex, in very good agreement with the data, as shown in the figure. A better agreement could be obtained also by adopting a steeper than Ballero et al. (2007a) IMF for the bulge, as discussed for Fig. 5, which would lower the $[\mathrm{O} / \mathrm{Mg}]$ ratio by $\sim 0.2$ dex. However, a steeper IMF for the bulge would not account for the stellar metallicity distribution nor for the mean metallicity in the bulge (see Ballero et al. 2007b; Pipino et al. 2008). Finally, part of the problems could reside in the still uncertain $\mathrm{Mg}$ yields.

\section{Discussion and conclusions}

We have reviewed stellar CNO abundances in the thin and thick disks and the bulge of the Galaxy. Because the bulge red giant stars envelopes are contaminated by first dredge-up, $\mathrm{CN}$ processed, material in their envelopes, we estimate their primordial $[\mathrm{C} / \mathrm{O}]$ ratios from $\mathrm{C}+\mathrm{N}$ minus a correction for the original nitrogen abundance $(\sim 0.1 \mathrm{dex})$. We showed that with careful measurements this method can give $[\mathrm{C} / \mathrm{O}]$ ratios from red giants identical to unmixed dwarf/turnoff stars in the thin and thick disks.

From our review of such $\mathrm{CNO}$ abundances in Galactic bulge red giants we find a large increase in the $[\mathrm{C} / \mathrm{O}]$ ratio $(>0.5 \mathrm{dex})$ that is tightly correlated with metallicity between $[\mathrm{O} / \mathrm{H}]=-0.1$ and +0.4 dex. This observed change in bulge $[\mathrm{C} / \mathrm{O}]$ ratio can be reproduced with bulge chemical evolution models that incorporate yields from massive stars including the effects of metallicity-dependent stellar winds and rotation; in particular our models would best match the observations with mass-loss rates intermediate between the values adopted by M92 and MM02. This supports the idea that the decline in $[\mathrm{O} / \mathrm{Mg}]$ and $[\mathrm{O} / \mathrm{Fe}]$ seen in the bulge near solar metallicity is due a decline in oxygen yields in massive stars by metallicity-dependent winds, as suggested by McWilliam \& Rich (2004) and McWilliam et al. (2008). This is important because it resolves the apparent discrepancy in interpretation of the bulge formation timescale based on simplistic interpretation of the bulge trends of $[\mathrm{Mg} / \mathrm{Fe}]$ and $[\mathrm{O} / \mathrm{Fe}]$. These conclusions on the bulge $[\mathrm{C} / \mathrm{O}]$ ratio support a rapid bulge formation timescale $(<1 \mathrm{Gyr})$.

To better match the slope of the bulge $[\mathrm{C} / \mathrm{O}]$ ratio with $[\mathrm{O} / \mathrm{H}]$ we found it necessary to interpolate the predicted stellar yields, rather than employ a step function change in yield at solar metallicity. We employ the same interpolation technique for our models of the thin disk $[\mathrm{C} / \mathrm{O}]$ trend with $[\mathrm{O} / \mathrm{H}]$, and we find a remarkably good correspondence between our model results and the thin disk observations. In particular, we find that the $[\mathrm{C} / \mathrm{O}]$ slope in the thin disk is best matched with the yields of MM02. In our models approximately $50 \%$ of the thin disk carbon comes from low mass stars. 
M08 found that the bulge and thick disk $[\mathrm{O} / \mathrm{Fe}]$ versus $[\mathrm{Fe} / \mathrm{H}]$ trends are identical; our new oxygen results in MFR09 agree with this conclusion. Remarkably, the data examined here shows that the $[\mathrm{C} / \mathrm{O}]$ versus $[\mathrm{O} / \mathrm{H}]$ locus in the thick disk is also practically identical to the trend in the Galactic bulge. We assume that the common trends in these two systems is due to higher star formation rates, and faster formation timescales, than occurred in the thin disk. However, the higher $[\mathrm{Mg} / \mathrm{Fe}]$ in the bulge than the thick disk (FMR07) is evidence that the bulge formed more quickly. Still, while the bulge and thick disk probably formed fairly rapidly it is remarkable that these two systems have such different mean metallicities $(\Delta[\mathrm{Fe} / \mathrm{H}] \sim 0.5$ dex $)$.

As mentioned earlier, Ballero et al. $(2007 \mathrm{a}, \mathrm{b})$ employed a shallow IMF (i.e. more high mass stars) in order to fit the bulge stellar metallicity distribution as well as the bulge mean metallicity with a rapid formation timescale. Similarly, it may be that while the thick disk and the bulge both formed fairly quickly (at least relative to the thin disk), metallicities may largely reflect different SF efficiencies (lower in the thick disk, Chiappini 2009), and/or different IMFs. In other words, our best model for the bulge cannot reproduce the mean metallicity and the metallicity distribution of the thick disk stars.

While our models naturally explain the increase in the $[\mathrm{C} / \mathrm{O}]$ ratio to higher metallicity, there is a plateau at low $[\mathrm{O} / \mathrm{H}]$ in the bulge data; a similar plateau is also seen in our models, but $\sim 0.2$ dex lower than the observations. While this plateau problem could, in principle, be solved with a change in the bulge IMF this would lead to a conflict with the observed mean metallicity and the rapid bulge formation timescale indicated by $[\mathrm{Mg} / \mathrm{Fe}]$; thus, we rejected appeals to the IMF to solve the problem. The under-predicted plateau suggests to us that there is a missing source of carbon in our models, and/or an under-production of oxygen. Extremely metal poor stars $\left(Z \sim 10^{-8}\right)$ can be very fast rotators, suffer heavy mass loss and produce that extra $\mathrm{C}$ (Hirschi 2006; Chiappini et al. 2006), but to explain the plateau such a production should extend also to stars with higher metallicities. Another cause, although prosaic, can be an incomplete stellar evolution physics, wind physics, or error in the adopted ${ }^{12} \mathrm{C}(\alpha$, $\gamma)^{16} \mathrm{O}$ rate. We highlight an alternative explanation: stripping of the envelopes of massive stars in binary systems through Roche lobe overflow, which would work even at low metallicity where winds don't. We presume that this form of envelope stripping would also result in an increase in carbon yield at the expense of oxygen. Finally, the disagreement between theory and observations could be ascribed to observational uncertainties.

Acknowledgements. G.C. acknowledges financial support from the Fondazione Cassa di Risparmio di Trieste. G.C., F.M. and C.C. acknowledge financial support from PRIN2007-MIUR (Italian Ministry of University and Research). Prot.2007JJC53X-001. C.C. acknowledges financial support from the Swiss National Foundation (SNF). F.M. thanks R. M. Rich for many illuminating discussions. We thank the referee, Martin Asplund, for his careful reading and his suggestions.

\section{References}

Allende Prieto, C., Lambert, D. L., \& Asplund, M. 2001, ApJ, 556, 63 Andersson, H., \& Edvardsson, B. 1994, A\&A, 290, 590

Arnett, D. 1991, ApJ, 383, 295

Asplund, M., Grevesse, N., \& Sauval, A. J. 2005, ASPC, 336, 25A

Ballero, S. K., Matteucci, F., Origlia, L., \& Rich, R. M. 2007a, A\&A, 467, 123

Ballero, S. K., Kroupa, P., \& Matteucci, F. 2007b, A\&A, 467, 117

Becker, S. A., \& Iben, I. 1979, ApJ, 232, 831

Bensby, T., \& Feltzing, S. 2006, MNRAS, 367, 1181

Bensby, T., \& Feltzing, S. 2009, Proceedings of Science, in press [arXiv:0809.4249]

Carigi, L., Peimbert, M., Esteban, C., \& García-Rojas, J. 2005, ApJ, 623, 213

Chiappini, C. 2009, IAU Symp., ed. J. Andersen, J. Bland-Hawthorn, \& B. Nordström, 254, 191

Chiappini, C., Matteucci, F., Beers, T. C., \& Nomoto, K. 1999, ApJ, 515, 226

Chiappini, C., Romano, D., \& Matteucci, F. 2003a, MNRAS, 339, 63

Chiappini, C., Matteucci, F., \& Meynet, G. 2003b, A\&A, 410, 257

Chiappini, C., Hirschi, R., Meynet, G., et al. 2006, A\&A, 449, L27

Cunha, K., \& Smith, V. V. 2006, ApJ, 651, 491

Fabbian, D., Asplund, M., Carlsson, M., \& Kiselman, D. 2006, A\&A, 458, 899

Fabbian, D., Nissen, P. E., Asplund, M., Pettini, M., \& Akerman, C. 2009, A\&A, 500, 1143

François, P., Matteucci, F., Cayrel, R., et al. 2004, A\&A, 421, 613

Fulbright, J. P., McWilliam, A., \& Rich, R. M. 2007, ApJ, 661, 1152

Greggio, L., \& Renzini, A. 1983, A\&A, 118, 217

Grevesse, N., \& Sauval, A. J., 1998, Space Sci. Rev., 85, 161

Gustafsson, B., Karlsson, T., Olsson, E., Edvardsson, B., \& Ryde, N. 1999, A\&A, 342, 426

Hirschi, R. 2006, Rev. Mod. Astron., 19, 101

Hirschi, R., Meynet, M., \& Maeder, A. 2005, A\&A, 433, 1013

Iben, I. 1964, ApJ, 140, 1631

Iben, I. 1967, ARA\&A, 5, 571

Johnson, J. A., Gaudi, B. S., Sumi, T., Bond, I. A., \& Gould, A. 2008, ApJ, 685, 508

Karakas, A., \& Lattanzio, J. C. 2007, PASA, 24, 103

Kroupa, P. 2002, Science, 295, 82

Lambert, D. L., \& Ries, L. M. 1981, ApJ, 248, 228

Lecureur, A., Hill, V., Zoccali, M., et al. 2007, A\&A, 465, 799

Matteucci, F., \& Greggio, L. 1986, A\&A, 154, 279

Maeder, A. 1992, A\&A, 264, 105

McWilliam, A., \& Rich, R. M. 2004, ApJS, 91, 749

McWilliam, A., Matteucci, F., Ballero, S., et al. 2008, AJ, 136, 367

Meléndez, J., Asplund, M., Alves-Brito, A., et al. 2008, A\&A, 484, L21

Meynet, G., \& Maeder, A. 2002, A\&A, 390, 561

Mishenina, T. V., Bienaymé, O., Gorbaneva, T. I., et al. 2006, A\&A, 456, 1109

Nissen, P. E., \& Edvardsson, B. 1992, A\&A, 261, 255

Nissen, P. E., Primas, F., Asplund, M., \& Lambert, D. L. 2002, A\&A, 390, 235

Paczyński, B. 1967, Acta Astronomica, 17, 355

Pipino, A., Matteucci, F., \& D'Ercole, A. 2008, in Formation and Evolution of

Galaxy Bulges, Proceedings of the International Astronomical Union, IAU Symp., 245, 19

Pipino, A., Chiappini, C., Graves, G., \& Matteucci, F. 2009,

[arXiv:0904.2168]

Rich, R. M., \& Origlia, L. 2005, ApJ, 634, 1293

Rich, R. M., Origlia, L., \& Valenti, E. 2007, ApJ, 665, L119

Ryde, N., Edvardsson, B., Gustafsson, B., et al. 2009, A\&A, 496, 701

Scalo, J. M. 1986, FCPh, 11, 1

Schönrich, R., \& Binney, J. 2009, MNRAS, 396, 203

Scott, P., Asplund, M., Grevesse, N., \& Sauval, A. J. 2009, ApJ, 691, L119

Tinsley, B. M. 1979, ApJ, 229, 1046

van den Hoek, L. B., \& Groenewegen, M. A. T. 1997, A\&AS, 123, 305

Woosley, S. E., \& Weaver, T. A. 1995, ApJ, 101, 181

Zoccali, M., Lecureur, A., Barbuy, B., et al. 2006, A\&A, 457, 1 